\title{
EDITORIAL
}

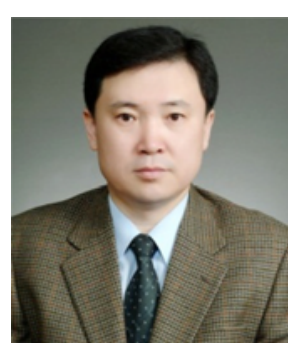

\section{Special Issue on Implantable Neural Interface}

\author{
Jin Woo Chang
}

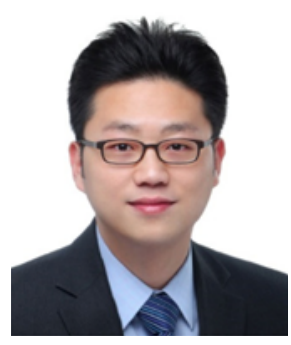

Sang Beom Jun

(C) The Korean Society of Medical \& Biological Engineering and Springer 2016

For more than a hundred years, since people found that brain is the very centralized organ processing behaviors, thinking, emotions, memory and learning, researchers in neuroscience fields have tried to understand the underlying mechanisms of the brain using various techniques. As a result of these efforts, it has become possible to understand the basic principles of brain functions and the neural circuits. Based on these knowledge, various treatments of neurological diseases have been also developed and clinically being utilized including the neuropharmacological therapies, surgical operations and neuromodulations for stimulation or suppression of specific brain regions. Especially, during the past decade, deep brain stimulation (DBS) has become one of the most successful neuromodulation methods to dramatically improve the motor functions of patients with Parkinson's disease, essential tremor, obsessive-compulsive disorder and primary dystonia. In addition, there are several reports that neuromodulation

Jin Woo Chang

Department of Neurosurgery, Yonsei University College of Medicine, Seoul, Republic of Korea

Tel : +82-2-2228-2150

E-mail : jchang@yuhs.ac

Sang Beom Jun $(\varangle)$

Department of Electronics Engineering, Ewha Womans University, Seoul, Republic of Korea

Tel : +82-2-3277-3892 / Fax : +82-2-3277-3494

E-mail :juns@ewha.ac.kr could be effective to treat other neurological diseases such as epilepsy, chronic pain, depression, Tourette syndrome, addictions, and Alzheimer disease and so forth. Therefore, the technologies related to the implantable neuromodulation systems are developing very rapidly.

Despite the effectiveness of current neuromodulation therapies such as DBS, cochlear implant and artificial retina, however, there still exist unclear mechanisms to be investigated. Therefore, a number of researchers in various fields are trying to improve current technologies and develop a novel approaches. This special issue on "Implantable Neural Interface" aims to introduce the cutting-edge technologies related to implantable neural prosthetic systems from various fields of engineering, science, and medicine. The technologies include electrodes, signal processing, whole prosthetic system design, wireless communications, electrochemical detection of neurotransmitters, closed neuromodulation strategies, and so on.

The paper entitled "A Review of Electrodes for the Electrical Brain Signal Recording" by Im and Seo provides a brief review of the various types of electrodes for recording electrical brain signals [1]. It includes implantable electrodes for detection of electrocorticogram $(\mathrm{ECoG})$, local field potential (LFP), and spike activity as well as noninvasive electrodes mostly for electroencephalogram (EEG). They reviewed the detailed fabrication procedure and the characteristics of each electrode type. The material information of electrodes and 
insulators will give a practical guidance to the researchers who attempts to use implantable neural interfaces. The paper entitled "Neural signal processing for closed-loop neuromodulation" by Cha et al. provides a timely review of state-of-arts neuromodulation methods using closed-loop feedback system [2]. Nowadays, the closed-loop neuromodulation attracts a numerous attentions from researchers and manufacturers of neuroprosthetic systems. Recently, it has been shown that the closed-loop system can provide effective solutions for treating a various neurological diseases such as Parkinson's diseases, epilepsy, chronic pain, and so forth. This paper reviews the signal processing techniques which are relevant to realize the successful closed-loop therapeutic systems.

The detection of neural activity is performed typically by electrical means due to the ease of system implementation. It is also because the ionic flow accompanying the neural signal can be easily measured as electrical voltage or current. However, recently the detection of neurotransmitter has been proposed and shown to be feasible to detect a specific species of neurotransmitters. The article entitled "Electrochemical detection of neurotransmitters: Toward synapse-based neural interfaces" by Jeon et al. introduces the principles of two major approaches for electrochemical neurotransmitter detection, amperometry and fast-scan cyclic voltammetry (FSCV) [3]. They also suggest the possibility that these electrochemical methods can be employed as a novel neural interfaces which is capable of detecting the neurotransmitter release in the synapses.

In this special issue, there are two review papers focused on the neural interfaces for animals. The first article entitled "Neuromodulation methods for animal locomotion control" by Cho et al. reviewed an intriguing topic of controlling animals' behavior by means of neuromodulation [4]. They reviewed the two major underlying principles for controlling animals' behaviors. One is to stimulate dopaminergic mesolimbic pathways or amygdala region for operant reward or fear conditioning. The other is to directly modulate motor functions by stimulating nigrostriatal pathways. They also reviewed the detailed stimulation parameters for electrical and optogenetic neuromodulations. The second paper is entitled "Development of wireless neural interface system" by $\operatorname{Im}$ et al. [5]. This article introduces a wireless neural interface system (WNIS) for animal experiments. They implemented a MCU-based wireless system which is capable of multi-channel neural recording and stimulation. This system is equipped with Bluetooth communication module to wirelessly record LFP signals from spinal nerves and control bladder contractions. The system concepts can be applied to facilitate neuroscience research with freely moving animals.

The review article entitled "Liquid crystal polymer-based neural prosthetic devices" by Gwon et al. introduces a series of neural prosthetic systems using liquid crystal polymer (LCP) [6]. To date, clinical implantable neural prosthetic systems (i.e. cochlear implant, deep brain stimulation system) are composed of metallic packages and molded multichannel electrode arrays. The authors have been engineering polymerbased monolithic implantable neural prostheses using LCP as a substrate as well as a package. Since this polymer has been proven biocompatible, inert, and highly water resistant, it is suitable for encapsulation material instead of metallic packages. The LCP-based monolithic integration of electrodes and packaging is advantageous in terms of compatibility with microfabrication, low material and low fabrication cost, MRI compatibility, and so forth. In this review, the current technological status of LCP-based neural prostheses are provided including LCP-base electrode arrays, packaging methods, cochlear implant system, and artificial retina.

As a last topic of the current issue, the original article entitled "Three-dimensional neural cell construct for implantable neural interface" by Lee et al. is included [7]. Different from other articles, it describes in vitro three-dimensional coculturing method of neurons and astrocytes in order to mimic more realistic conditions than two-dimensional cultures. Even though this study might seem far from the implantable neural interfaces, there have been attempts to assemble neuron-cultured constructs on the implantable electrodes to trap the neurons near electrode sites and therefore maintain the stable connection between electrode and neurons in the brain after the electrode implantation. Therefore, this study can provide not only a methodology for in vitro studies but also a noble strategy to circumvent reactive glial response to the implanted electrode and to form long term stable neural interfaces.

As the converged technologies are required for successful development of implantable neural prostheses, the articles included in this special issues are contributed by various collaborative research teams of engineering, science, and medicine. We hope that the information provided in the review and the original articles are helpful for the researchers as well as people who are interested in this field. Finally, we would like to appreciate the efforts of the authors who contributed to this special issue. We would also like to thank to the devoted support of the Managing Editor (Prof. Jae Sung Lee) and the Editor-in-Chief (Prof. Sang-Hoon Lee) of Biomedical Engineering Letters.

\section{REFERENCES}

[1] Im C, Seo J. A review of electrodes for the electrical brain signal recording. Biomed Eng Lett. 2016; 6(3):104-12. 
[2] Cha KS, Yeo D, Kim KH. Neural signal processing for closedloop neuromodulation. Biomed Eng Lett. 2016; 6(3):113-22.

[3] Jeon J, Hwang I, Chung TD. Electrochemical detection of neurotransmitters: toward synapse-based neural interfaces. Biomed Eng Lett. 2016; 6(3):123-33.

[4] Cho YK, Kim S, Jung HH, Chang JW, Kim YJ, Shin HC, Jun SB. Neuromodulation methods for animal locomotion control. Biomed Eng Lett. 2016; 6(3):134-47.

[5] Im C, Koh CS, Park HY, Shin J, Jun SB, Jung HH, Ahn JM,
Chang JW, Kim YJ, Shin HC. Development of wireless neural interface system. Biomed Eng Lett. 2016; 6(3):164-71.

[6] Gwon TM, Kim C, Shin S, Park JH, Kim JH, Kim SJ. Liquid crystal polymer (LCP)-based neural prosthetic devices. Biomed Eng Lett. 2016; 6(3):148-63.

[7] Lee W, Shim S, Park JH, Kim SJ. A three-dimensional neural cell construct for implantable neural interface. Biomed Eng Lett. 2016; 6(3):172-80. 